\title{
Richtig essen (und trinken)
}

\section{Hans Stalder}

Prof. Dr. med., Mitglied der Redaktion

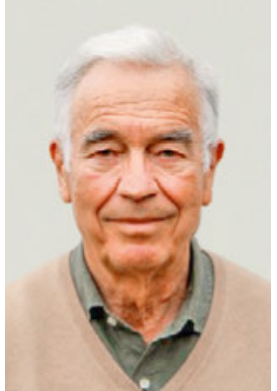

Literatu

1 Nissen SE. U.S. dietary guidelines: an evidencefree zone. Ann Intern Med. 2016;164:558-9. Kiefer B. Nutrition: le renversement des certirenversement des certitudes (dt. Übers. Erkennt niswandel). Revue médicale suisse. 2017;13:1632 Dehghan M, Mente A, Zhang X, et al. ProspecZhang X, et al. Prospectije Urban Rural Epidemiology (PURE) study investigators. Associations of fats and carbohydrate intake with cardiovascular diseas cardiovascular dise and mortality in 18 countries from five continents (PURE): a prospective cohort study. Lancet. 2017 Aug 28. pii: S0140-6736(17)32252-3. doi: 101016/s0140 doi: 10.1016/s01406736(17)32252-3

Topiwala A, Allan CL, Valkanova $\mathrm{V}$, et al. Moderate alcohol consumption as risk facto for adverse brain outfor adverse brain outcomes and cognitive decline: longitudinal cohort study. BMJ. 2017;357:j2353. doi 10.1136/bmi.j2353. (1.1136/bmj.j235s. Estruch RI, Ros E, Sala Salvadó J, Covas MI, et al. Primary prevention of cardiovascular disease with a Mediterranean diet. N Engl ranean diet. Nengl Med. 2013,368.1279-90.

6 Brillat-Savarin JA. Phy siologie Des $\mathrm{Ge}-$ schmacks: Oder, Physiologische Anleitung Zum Studium Der Tafelgenüsse. Den Pariser Gastronomen Gewidmet Von Einem Professor, Mitglied Vieler Gelehrten Gesellschaften. 1825 A Sautelet. Gabrielde Gosset ed. Paris.
In meiner Zeit als Assistenzarzt - das ist schon eine Weile her - spielte das Thema Diätetik eine grosse Rolle. Da noch keine Endoskopie in Sicht war, leitete der Professor der Gastroenterologie die Schule für Ernährung und Diätetik und predigte strikte Regeln wie beispielsweise die Sippy-Diät 1, 2 und 3- sie bestand aus faden Milchprodukten zur Ulcus-Behandlung- einer salzlosen Diät bei Herzinsuffizienz, fettfreier Ernährung bei Cholecystitis und einer Nullkaloriendiät bei Fettleibigkeit.

Als in der Folge stärker wirkende Medikamente auf den Markt kamen, waren die Spitäler eher bemüht, gute Mahlzeiten auf den Tisch zu bringen.

In den 70ern kam dann plötzlich wieder der Diätenkult auf die Agenda, allerdings eher im Bereich der Präventivmedizin. Nachdem eine multinationale Kohortenstudie aufzeigte, dass der Konsum von gesättigten Fetten mit der Entwicklung von Arteriosklerose, das heisst mit kardiovaskulären Erkrankungen in Verbindung zu bringen war, fühlte sich alle Welt genötigt, Fette wegzulassen und den Energieverlust durch Kohlenhydrate auszugleichen. Eier wurden verpönt, denn sie enthalten Cholesterin! Hätten die Autoren jedoch Frankreich mit in ihre Studie aufgenommen, so wären sie auf das French miracle gestossen, das heisst, dass der Verzehr von Fetten nicht unbedingt zu einem Anstieg der Herz-Kreislauf-Erkrankungen führen muss. Und schon war die Mittelmeerdiät geboren!

Und heute? Nach Bertrand Kiefer [2] kam es erneut zu einem «Erkenntniswandel». Eine von mehr als 200 Forschern mit über 133000 Personen in 18 Ländern durchgeführte Studie machte deutlich, dass Herz-KreislaufErkrankungen mit dem Konsum von Kohlenhydraten [3] in Verbindung stehen. Der Verzehr von tierischenFetten soll also gesund sein! Und schon steht die Hypothese im Raum, dass uns der in den letzten Jahrzehnten erfolgte Wechsel von Fetten zu Kohlenhydraten dick gemacht habe [1] ...

Ähnliches geschieht beim Alkohol: Lange Zeit wurde uns versichert, ein moderater Alkoholkonsum sei gut für die Gesundheit (dank der berühmten J-Kurve). Nun postuliert ein jüngst veröffentlichter Artikel, dass bereits geringste Mengen von Alkohol zu anatomischen und funktionellen Veränderungen im Gehirn führen [4].
Wie kommt es dazu? Die Antwort scheint mir nicht so schwierig: Alle diese Empfehlungen basieren auf Kohortenstudien. In der ernährungsbasierten Primärprävention gibt es nur wenige randomisierte Prospektivstudien und vor allem keine doppeltblinden (letztere sind in diesem Bereich nicht realisierbar). Nun wissen wir aber bereits seit der Revision der Empfehlungen in der postmenopausalen Hormonbehandlung, dass bei ausschliesslich auf Kohortenstudien basierenden Empfehlungen Vorsicht geboten ist. Diese Unsicherheitsfaktoren führten zu einer Verbreitung von Diätempfehlungen ohne die geringste wissenschaftlich fundierte Basis - vegetarisch, vegan, glutenfrei, Bio-, all dies füllt die Bücherregale und wird im Netz zur Freude der entsprechenden Nahrungsmittellobbyisten verbreitet!

Nur in Spanien wurde eine randomisierte Prospektivstudie durchgeführt. Sie machte deutlich, dass die zusätzliche Verwendung von Olivenöl oder Nüssen dasAuftreten von Herz-Kreislauf-Erkrankungen bei Risikopersonen senkte [5]. Aber Achtung: Bevor wir jetztalle Nüsse und Olivenöl in unseren Speiseplan aufnehmen, sollten wir daran denken, dass wir nicht alle Risikopersonen oder gar Spanier sind!

Was also können wir empfehlen? Zunächst einmal, dass unsere Präventologen vorsichtig umgehen mit Empfehlungen [1]! Dann wäre anzumerken, dass es sich bei jenen Studien [3, 4], die einen "Erkenntniswandel» [2] bewirkten, ebenfalls nur um Kohortenstudien handelte. Also keine Aufregung! Das Positive an diesen widersprüchlichen Ergebnissen und fehlenden randomisierten Prospektivstudien ist, dass wir so wenigstens in aller Ruhe das essen und trinken können, was uns schmeckt. Vielleicht sollten wir uns jedoch an den Grundsatz des alten Jean Anthelme Brillat-Savarin erinnern (besser bekannt durch seinen Satz «Sag mir was $\mathrm{du}$ isst und ich sage dir, wer du bist»), der in seinem Buch «Physiologie du goût, ou méditations de gastronomie transcendante» anmerkte, dass «diejenigen, die sich vollfressen oder besaufen, nicht wissen, wie man trinkt oder isst» [6]. Und wenn Sie (oder Ihre Kranken) fettleibig sind? Da gibt es leider nur eine Diät, die wie die damaligen allerdings recht unangenehm ist: $\mathrm{FdH}$ («Friss die Hälfte»)! 